\title{
EEG-BASED EVENT DETECTION USING OPTIMIZED ECHO STATE NETWORKS WITH LEAKY INTEGRATOR NEURONS
}

\author{
Sudhanshu Ayyagari ${ }^{1,2}$, Richard Jones ${ }^{1,2,3}$, Stephen Weddel1 ${ }^{1,2}$, \\ ${ }^{1}$ Electrical \& Computer Engineering, University of Canterbury, ${ }^{2}$ Christchurch Neurotechnology Research \\ Programme, ${ }^{3}$ New Zealand Brain Research Institute, Christchurch, New Zealand.
}

INTRODUCTION: Long-haul truck drivers, train drivers and commercial airline pilots routinely experience monotonous and extended driving periods in a sedentary position, which has been associated with drowsiness, microsleeps, and, consequently, serious accidents. Microsleeps are brief involuntary events of lapses in attention or responsiveness, associated with events such as prolonged eye closure which usually last from $0.5-15 \mathrm{~s}$ [1]. Consequently, the detection and preferable prediction of the microsleeps in subjects, especially those working in these high-risk occupations, is very important to workplace safety. The current study aims at developing a microsleep detector using the novel recurrent neural network architecture of an echo state network (ESN) and represents progression of our research from previous methods $[1,2]$.

METHODS: Artificial events were used to validate a prototype EEG-based microsleep detection system based around an echo state network (ESN) and a linear discriminant analysis (LDA) classifier. The artificial events comprised infrequent 2-s long bursts of $15 \mathrm{~Hz}$ sinusoids superimposed on prerecorded 16-channel EEG data which provided a means of determining and optimizing the accuracy of overall classifier on 'gold standard' events. The performance of this system was tested on different signal-to-noise amplitude ratios (SNRs) ranging from 16 down to 0.03 (16, 3, 1, 0.3, 0.03 ). Artificial datasets were used primarily to determine the detection performance of various detection systems/ configurations on a gold-standard dataset for which the events were precisely known. Six of the 2 -s segments had
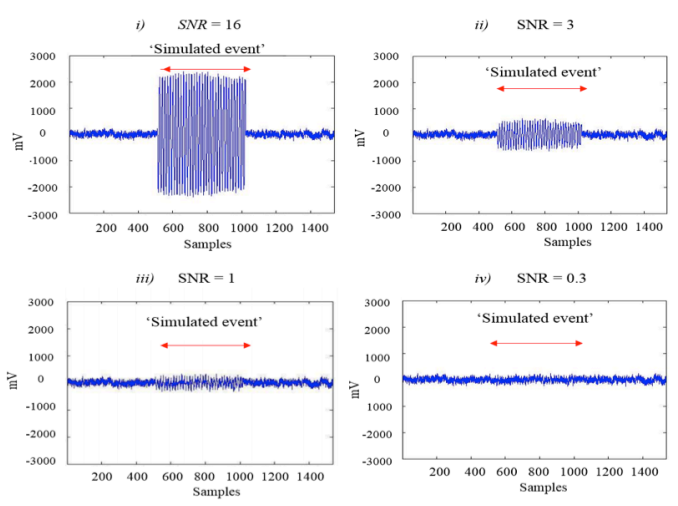

Fig. 1: An artificial event (15 Hz sinusoidal burst) superimposed on the ambient EEG. the $15 \mathrm{~Hz}$ sinusoidal bursts, equating to $2 \%$ of the time being events and $98 \%$ non-events. This highly unbalanced dataset parallels that of the relatively rare, albeit surprisingly high, occurrence of microsleeps.

RESULTS: Results from several feature selection/reduction and pattern classification modules indicated that training the classifier using a leaky-integrator neuron ESN [3] structure yielded highest classification accuracy. All feature selection/reduction modules led to a high classification performance on unbalanced data at SNRs 16 and 3. For datasets with a low SNR of 0.3, the leaky-neuron ESN, resulted in a phi correlation of 0.92 compared to 0.37 that employed principal component analysis (PCA). On the same datasets, other classifiers such as LDA and sigmoidal ESNs using PCA performed weakly with a correlation of 0.05 and 0 respectively.

DISCUSSION \& CONCLUSIONS: ESNs with leaky integrator neurons provided consistent and encouraging results, suggesting that ESNs with leaky neuron architectures have superior pattern recognition properties. This, in turn, may reflect their superior ability to exploit differences in state dynamics and, hence, provide superior temporal characteristics in learning.

\section{REFERENCES:}

${ }^{1}$ P. R. Davidson, R. D. Jones, and M. T. R. Peiris, (2007) IEEE Trans Biomed Eng, 54:832-9.

${ }^{2}$ M.T.R. Peiris, P.R. Davidson, P.J. Bones, and R.D. Jones, (2011) Journal of Neural Engineering, 8:1-15.

${ }^{3}$ M. H. Jaeger, M. Lukoševičius, and D. Popovici, (2007) Journal of Neural Networks, 20: 335-52. 